\title{
MAMMIFÈRES AQUATIQUES ET SEMI-AQUATIQUES INTRODUITS EN FRANCE. RISQUES ET CONSÉQUENCES.
}

\author{
F. MOUTOU
}

CNEVA Alfort, B.P. 67, 94703 Maisons-Alfort

et Société Française pour l'Etude et la Protection des Mammifères.

\section{RÉSUMÉ}

La faune mammalienne française, qui compte un peu plus d'une centaine d'espèces, héberge maintenant 7 espèces aquatiques ou semi-aquatiques introduites. II faut y ajouter le castor canadien, récemment éliminé. Ces 8 espèces appartiennent à 7 familles et à 3 ordres zoologiques différents. Les risques associés à la présence de ces animaux sont essentiellement d'ordre économique, écologique et sanitaire. Quelques exemples sont développés pour concrétiser ces cas de figure.

\section{AQUATIC AND SEMI-AQUATIC MAMMALS INTRODUCED INTO FRANCE. RISKS AND CONSEQUENCES.}

\section{SUMMARY}

The French mammal fauna comprises about a hundred species, 7 of which are aquatic or semi-aquatic introduced species. The Canadian beaver must be added to this list, but it has recently been eradicated. These 8 species belong to 7 families and 3 mammalian orders. The risks linked to these animals are mainly of three categories: economical, ecological and sanitary. A few examples are given to illustrate those situations.

\section{INTRODUCTION}

Comme l'a présenté H. MAURIN (1997), la faune mammalienne française est composée d'un certain nombre d'espèces aquatiques ou semi-aquatiques. Parmi cet ensemble, on note la présence de huit espèces introduites, allochtones, appartenant à 7 familles différentes réparties en trois ordres. Quatre sont issues d'Amérique du Nord, une d'Amérique du Sud et les trois dernières sont asiatiques. Le tableau I résume ces données. II se trouve que ces espèces n'ont pas été introduites qu'en France. De ce fait, les conséquences de leur présence sont connues, en partie, dans d'autres pays, même si toutes les situations ne peuvent pas être toujours comparées directement.

Cette présentation va donc consister à illustrer certains des risques connus associés à ces mammifères, récemment introduits pour la plupart dans notre pays. Le tableau II rassemble leurs statuts juridiques actuels et les risques reconnus, organisés en trois catégories : économique, écologique et sanitaire. II resterait un quatrième type de risque à évoquer pour être complet, le risque social ou l'idée que l'on peut se faire de la présence de telle ou telle espèce. $\mathrm{lci}$, les choses sont nettement plus subjectives et dépendent de nombreux paramètres difficiles à quantifier. Nous essayerons, dans la mesure du possible, de rester dans le domaine du quantifiable. Après l'analyse globale de ce tableau II, nous illustrerons, à partir de quelques exemples, des cas particuliers de risques rencontrés avec ces espèces. 
Tableau I

Les mammifères aquatiques et semi-aquatiques introduits en France. Systématique d'après WILSON et REEDER (1993).

Table I

Aquatic and semi-aquatic mammals introduced in France. Systematics following WILSON and REEDER (1993).

\begin{tabular}{|l|l|l|l|l|}
\hline \multicolumn{1}{|c|}{ Nom français } & Nom scientifique & \multicolumn{1}{c|}{ Famille } & \multicolumn{1}{c|}{ Ordre } & \multicolumn{1}{c|}{ Origine } \\
\hline Rat musqué & Ondatra zibethicus & Muridés & Rongeurs & Amérique du Nord \\
\hline Surmulot & Rattus norvegicus & Muridés & Rongeurs & Asie \\
\hline Ragondin & Myocastor coypus & Myocastoridés & Rongeurs & Amérique du Sud \\
\hline Castor canadien & Castor canadensis & Castoridés & Rongeurs & Amérique du Nord \\
\hline Raton laveur & Procyon lotor & Procyonidés & Carnivores & Amérique du Nord \\
\hline Chien viverrin & $\begin{array}{l}\text { Nyctereutes } \\
\text { procyonoides }\end{array}$ & Canidés & Carnivores & Extrême-Orient \\
\hline Vison d'Amérique & Mustela vison & Mustélidés & Carnivores & Amérique du Nord \\
\hline Hydropote de Chine & Hydropotes inermis & Cervidés & Artiodactyles & Chine et Corée \\
\hline
\end{tabular}

\section{Tableau II}

Les risques associès aux mammifères aquatiques et semi-aquatiques introduits en France. G : gibier selon l'arrêté ministériel du 16 juin 1987 ; $N$ : nuisible selon l'arrêté ministériel du 30 septembre 1988 ; $P$ : risque potentiel ; $R$ : risque réel ; ? : manque d'information. Voir aussi le texte.

\section{Table II}

Risks linked to aquatic and semi-aquatic mammals introduced in France. $G$ : game species as in 16 June 1987 act ; $N$ : pest as in 30 September 1988 act ; P : potential risk ; R : real risk ; ? : lack of data. See text also.

\begin{tabular}{|l|l|c|c|c|}
\hline \multicolumn{1}{|c|}{ Espèces } & Statut & Economique & Ecologique & Sanitaire \\
\hline Rat musqué & G.N. & R & R & P \\
\hline Surmulot & Sans statut & R & R & R \\
\hline Ragondin & G.N. & R & R & R \\
\hline Castor canadien & Éliminé & $?$ & R & P \\
\hline Raton laveur & G.N. & $?$ & $?$ & P \\
\hline Chien viverrin & G.N. & $?$ & $?$ & P \\
\hline Vison d'Amérique & G.N. & R & R & $?$ \\
\hline Hydropote de Chine & ? & $?$ & $?$ & $?$ \\
\hline
\end{tabular}




\section{APPROCHE GLOBALE}

La lecture du tableau II peut faire ressortir quelques points communs à l'ensemble des espèces, sachant que les exemples du prochain paragraphe pourront développer certains de ces aspects.

\section{Statut}

On peut noter la rapidité avec laquelle la plupart de ces espèces ont obtenu un statut juridique en France. La première capture de chien viverrin authentifiée date de 1979 (DUCHENE et ARTOIS, 1988) et depuis, l'espèce est restée fort discrète. Inversement, l'hydropote de Chine est présent, en nature, en Haute-Vienne depuis environ les années 1960 et il a seulement suscité un arrêté préfectoral de protection (SAND et KLEIN, 1995). Le castor canadien, après quelques années de liberté dans I'Yonne, à la suite d'une évasion d'un parc animalier, a été éliminé dans les années 1980 (MAURIN et al., 1994). Le plus ancien des introduits, le rat surmulot, n'a pas de statut juridique en France. La jurisprudence qui assimilerait à un gibier non chassable l'hydropote ne semble pas devoir s'appliquer aux petits rongeurs (ANONYME, 1994).

\section{Risques}

II faut bien admettre que les risques réels liés à la présence de ces espèces ne sont pas simples à quantifier. Le surmulot et l'hydropote sont probablement à l'opposé sur une échelle de valeurs, complexe à établir, mais certainement de grande amplitude.

Dans nos cités et nos habitations, le surmulot ou rat d'égout, extension extrême des milieux aquatiques, est responsable de divers dégâts. Sur les îlots proches des côtes, il est la cause de disparition de nombreux oiseaux nicheurs et son rôle de résenoir de bactéries du genre Leptospira impose une vaccination particulière aux égoutiers, pour qui la maladie correspondante, la leptospirose, est reconnue comme maladie professionnelle. Inversement, sur les deux communes où il semble présent, l'hydropote ne doit poser aucun problème particulier, comme cela est reconnu en Angleterre où sa répartition est pourtant plus grande (FARRELL et COOKE, 1991).

II faut encore préciser que l'intensité des problèmes est perçue à travers des biais difficiles à contourner. Actuellement, le rat musqué semble complètement occulté par le ragondin. Pourtant, sur le terrain, les deux espèces cohabitent. Le côté nouveau de la présence généralisée du plus gros ragondin a fait oublier à beaucoup l'existence du plus petit rat musqué, dont on néglige du même coup les éventuels dégâts (BURIN DES ROZIERS, 1990b; PIQUEMAL et TOUSSAINT, 1991b).

\section{EXEMPLES}

\section{Le ragondin}

Cette espèce est actuellement très présente dans tout le pays et peut-être surtout dans la presse agricole. Elle fait l'objet de nombreuses campagnes de destructions de la part des organismes spécialisés (MICOL, 1990, 1991; BURIN DES ROZIERS, 1990a; PIQUEMAL et TOUSSAINT, 1991a). Pourtant, si la typologie des dégâts est facile à résumer : dégâts aux structures, dégâts aux cultures et incidences sur l'environnement avec en particulier la question de la spécificité des méthodes de lutte, l'estimation quantitative de ces mêmes dégâts reste difficile. Un hectare de maîs suivi en 1988 dans les Deux-Sèvres, et où habitait une famille de 10 à 15 ragondins, a vu son rendement estimé baisser de $3,2 \%$ à cause des rongeurs (PIQUEMAL et TOUSSAINT, 1991a). Cela reste modeste. Une enquête, faite en Vendée en 1994, propose le chiffre de $15000 \mathrm{~F}$ de pertes par exploitation, mesurées sur les parcelles de maïs et de tournesol. Le protocole de l'enquête et l'échantillonnage ne sont pas connus.

Inversement, le coût des méthodes de lutte est plus simple à obtenir. Trois méthodes sont possibles. Le tir est autorisé, puisque le ragondin est classé gibier et peut être classé nuisible. Comme ni l'usage de sa fourrure ni la consommation de sa viande ne représentent une 
forte incitation, les pratiques les plus usuelles sont la lutte chimique et le piégeage. La lutte chimique à base d'anticoagulants pose le problème du devenir des produits dans l'environnement. Le piégeage, à l'aide de cages, en surface, réduit les risques liés à la capture d'une autre espèce. Dans l'ouest de la France, loutre (Lutra lutra) et ragondin cohabitent. Des pièges vulnérants ou immergés pourraient constituer une menace sérieuse pour une espèce comme la loutre, totalement protégée et surtout menacée à l'échelle du continent européen. Le coût des méthodes de lutte peut aller jusqu'à $700000 \mathrm{~F}$ par an, pour un département comme le Maine-et-Loire (MICOL, 1990). Le rapport avec l'estimation chiffrée des dégâts serait important à pouvoir établir, mais semble rarement réalisé.

Les risques sanitaires liés à la présence du ragondin sont de deux ordres : risque pour le bétail et risque pour la santé humaine. On sait maintenant que le ragondin peut héberger la forme adulte de la douve du foie (Fasciola hepatica), helminthe trématode parasite des bovins, et donc créer un réservoir secondaire. En cas de lutte contre cette maladie chez les bovins, il est important d'apprécier l'état de la population locale de ragondins vis-à-vis du parasite. Pour la santé humaine, deux maladies bactériennes sont essentiellement à craindre, la leptospirose et la tularémie, qui peuvent être transmises par l'espèce. La fasciolose, la maladie causée par la douve, peut, à l'occasion, aussi être une zoonose, c'est-à-dire transmissible à l'homme (ACHA et SZYFRES, 1989 ; MOUTOU, 1989, 1990).

\section{Le castor canadien}

Cette espèce n'aura fait qu'un bref séjour en liberté sur le sol français, dans le courant des années 1970 et jusqu'au milieu des années 1980. Au moins un couple reproducteur s'était échappé d'un parc animalier dans I'Yonne. Installés sur un plan d'eau voisin, ils avaient bâti une première hutte, et le groupe familial semblait prêt à faire souche, après une première reproduction réussie. Le problème était ici essentiellement écologique. Le retour du castor européen (Castor fiber) en France, et sur la Loire tout particulièrement, pouvait être entravé par le dynamisme connu de l'espèce nord-américaine. Le cas de la Finlande est là pour nous rappeler que le retour de l'espèce européenne (environ 200 animaux) y est très difficile face à une population forte de 6000 animaux de l'espèce allochtone (MACDONALD, 1995). Pour éviter ce problème, il a été décidé d'éliminer, en les capturant pour les donner à des parcs zoologiques, tous les animaux de la colonie de l'Yonne, environ une dizaine d'animaux à cette époque (MAURIN et al., 1994). L'opération s'est heurtée à un problème imprévu. La population riveraine du plan d'eau où les animaux s'étaient installés n'a pas compris le sens de la démarche et ne voulait pas que l'on retire "ses " castors. Le risque écologique de compétition entre espèces autochtone et allochtone était totalement étranger aux soucis immédiats des habitants, et les convaincre n'a pas été le moindre des problèmes de cette opération. $\mathrm{Ce}$ fameux risque social évoqué plus haut comprend ce type de difficulté, loin des préoccupations scientifiques, économiques ou sanitaires, mais néanmoins très conflictuel quand il est présent.

Notons enfin que le castor canadien est responsable d'une amibiase à Giardia, la giardiose, responsable de la " fièvre des castors" en Amérique du Nord mais non décrite en Europe. Les campeurs nord-américains semblent s'en méfier tout particulièrement (ACHA et SZYFRES, 1989).

\section{Raton laveur et chien viverrin}

Au niveau de cette revue, il est légitime d'associer ces deux espèces, tant les problèmes posés se ressemblent. Inversement, il faut bien admettre qu'au niveau biogéographique la présence simultanée en France d'un procyonidé nord-américain et d'un canidé propre à l'Extrême-Orient est une surprise. Les deux espèces doivent se côtoyer dans le département de l'Aisne (DUCHENE et ARTOIS, 1988). Les poids économique et écologique de ces deux espèces semblent encore modestes. L'aire de répartition occidentale du chien viverrin, de l'Ukraine à la France et à la Finlande, est pourtant plus large que son aire de répartition naturelle. Apparemment, il y occupe une niche écologique restée vacante jusque-là et son régime volontiers charognard, omnivore, éclectique, lui offre de nombreuses possibilités d'adaptation (KAUHALA et HELLE, 1995; MACDONALD, 1995). Tous les animaux actuels descendent 
d'environ 9000 individus relâchés autour des années 1930 et 1940 en Russie européenne. Le rôle actuel du chien viverrin dans l'épidémiologie de la rage est une conséquence non prévue, et sérieuse, de sa présence. L'espèce entretient maintenant une rage indépendante de celle du renard, de la Finlande (ANONYME, 1988) à l'Ukraine (CHERKASSKIY, 1985). La vaste campagne d'éradication de la rage, entreprise en Europe de l'ouest avec la vaccination orale des renards, a vraiment pris de l'ampleur à partir de la fin des années 1980. Les résultats en sont très spectaculaires. II faut espérer que, plus à l'est, le chien viverrin ne remettra pas en question ces résultats. Parallèlement, aux Etats-Unis mêmes, nous savons maintenant que des déplacements de ratons laveurs depuis la Floride vers la Virginie, pour des raisons cynégétiques, ont permis le développement d'une vaste épizootie de rage due à cette espèce. Elle atteint maintenant le Canada (TORRENCE et al., 1992 ; ANONYME, 1995). Dans les lots déplacés, des ratons laveurs en incubation de rage avaient été prélevés sans contrôles sanitaires.

Comme ces deux espèces sont maintenant présentes en France, il nous faut être vigilants, en particulier par rapport à la rage. Le problème est devenu européen.

\section{Vison américain}

Cette espèce illustre plutôt les aspects écologiques de l'introduction d'espèces allochtones, même si les visons peuvent à l'occasion créer des problèmes économiques, probablement ponctuels cependant. Avec le nord de l'Espagne, la façade atlantique française correspond à la dernière zone où se trouve en Europe occidentale, le vison européen (Mustela lutreola) (SAINT-GIRONS, 1994), qui semble en régression rapide (CAMBY, 1990). Dans ces conditions, la présence d'une espèce proche, adaptable, ne peut que représenter une pression supplémentaire pour le mammifère européen le plus menacé. Les capacités du vison américain sont maintenant bien connues et la compétition entre les deux espèces risque de se faire au détriment de la forme autochtone (MAIZERET, 1990 ; DUNSTONE, 1993). Le vison américain a aussi été suspecté de concurrence avec la loutre européenne, dans les régions où il avait été introduit. Cela semble moins probable, à cause de la différence de taille et de régime alimentaire. En Amérique du Nord, vison d'Amérique et loutre du Canada (Lutra canadensis) cohabitent largement. Les analyses les plus récentes écartent ce risque en Europe, en signalant toutes les menaces nettement plus immédiates qui pèsent sur la loutre (LAIDLER, 1982 ; MACDONALD et MASON, 1994).

Enfin, pour ne pas terminer sur une note trop pessimiste, on peut rappeler que le vison américain est un prédateur efficace du rat musqué aux Etats-Unis (PERRY, 1982 ; LINSCOMBE et al., 1982). En Europe, le même vison pourrait peut-être retrouver cette proie et participer à son contrôle. Ceci est plus un voeu qu'une hypothèse, dans l'état de nos connaissances.

\section{CONCLUSION}

Ce rapide survol des problèmes liés à la présence de mammifères aquatiques et semiaquatiques introduits en France n'est certainement pas différent de ce que d'autres groupes zoologiques pourraient nous apprendre. Ils confirment nos difficultés à contrôler les introductions. La mode de l'acclimatation des XVIIle et XIXe siècles se poursuit à la fin du $X X e$ siècle. Pourtant, les études préalables et les évaluations des introductions ne sont toujours pas faites, malgré les nombreux arguments qui s'accumulent pour demander plus de prudence et de discernement dans ces déplacements de faune (MAURIN et al., 1994). Les raisons réelles de ces introductions sont des plus diverses et parfois surprenantes. $\|$ y a les échappés de captivité, les espèces relâchées volontairement pour leur fourrure ou pour de fausses idées sur l'intérêt écologique d'introduire des animaux exotiques là où ils n'étaient pas présents. Cela correspond encore au mythe ancien de l'acclimatation, mais semble plus à lier à l'image que l'on se fait de la nature qu'à toute réalité ou connaissance objective. Le bilan chiffré de ce qui a déjà èté introduit ne se faisant pas, il y a toujours des volontaires pour proposer de nouvelles expériences. 
Face à des espèces introduites, les méthodes de contrôle ne sont pas si simples. Le Royaume-Uni a réussi à se débarrasser du rat musqué et du ragondin (GOSLING et BAKER, 1989, 1991) en combinant une forte pression de capture à des aléas climatiques favorables, comme des hivers froids, défavorables au ragondin. En France, aujourd'hui, de telles campagnes semblent bien improbables. Si on laisse s'installer une espèce, elle risque de s'implanter durablement, et aussi de devenir partie intégrante de notre patrimoine. II y aura alors rapidement des voix pour s'opposer à son éradication. C'est assez probable avec des mammifères.

Un point important est aussi à soulever. Les introductions d'espèces étant souvent faites sans étude préalable, parfois même illégalement, il n'y a presque jamais de précaution sanitaire qui y soit associée. Or, de plus en plus, on réalise maintenant que les déplacements d'espèces se prolongent par des déplacements et des introductions d'agents pathogènes allochtones, virus, bactéries, champignons ou parasites (MOUTOU, 1994). Dans le domaine de l'ichtyopathologie, les exemples sont nombreux, mais avec les mammifères aussi il existe des cas très concrets. II est probable que les conséquences les plus spectaculaires des introductions se situent à ce niveau.

Finalement, les mammifères aquatiques et semi-aquatiques introduits en France illustrent parfaitement les divers types de risques classiquement associés aux introductions.

Signalons enfin que le ragondin semble en forte régression sur toute son aire de répartition d'origine, alors qu'il apparaît en expansion en France et en Europe.

\section{BIBLIOGRAPHIE}

ACHA P.N., SZYFRES B., 1989. Zoonoses et maladies transmissibles communes à l'homme et aux animaux. 2e édition. Office International des Epizooties, Paris, $1065 \mathrm{p}$.

ANONYME, 1988. Situation de la rage en Finlande. Bull. O.I.E., 100, (5), 342-343.

ANONYME, 1994. Instruction PN/S2 N90/2 du 18 avril 1990 fixant le régime juridique applicable aux mammifères et oiseaux d'espèces non domestiques appartenant au patrimoine biologique national. Protection de la Nature, Faune et Flore $N^{\circ} 1454-1$. Direction des Journaux Officiels, Paris, 25-31.

ANONYME, 1995. La poussée vers le Nord de la rage du raton laveur. Dernières nouvelles. Bulletin du Centre de la Santé de la Faune, 3, (3), 4-5.

BURIN DES ROZIERS H., 1990a. Luttes contre le ragondin. La Défense des végétaux, 260-261, 46-48.

BURIN DES ROZIERS H., 1990b. Le rat musqué. La Défense des Végétaux, 260-261, 49-50.

CAMBY A., 1990. Le vison d'Europe. Encyclopédie des Carnivores de France, 13, SFEPM, Paris, $18 \mathrm{p}$.

CHERKASSKIY B. L., 1985. La rage en Union Soviétique. Informations Techniques des Services Vétérinaires "Pasteur et la Rage ", 92 à 95, 27S-30S.

DUCHENE M.J., ARTOIS M., 1988. Les carnivores introduits : chien viverrin (Nyctereutes procyonoides Gray, 1834) et raton laveur (Procyon lotor Linnaeus, 1758). Encyclopédie des Carnivores de France, 4 et 6, SFEPM, Paris, $49 \mathrm{p}$.

DUNSTONE N., 1993. The mink. Poyser, London, $232 \mathrm{p}$.

FARRELL L., COOKE A., 1991. Genus Hydropotes in CORBET G.B. and HARRIS S., The Handbook of British Mammals, Third Edition, 532-537, Blackwell, Oxford.

GOSLING L.M., BAKER S.J., 1989. The eradication of muskrats and coypus from Britain. Biol. J. Linn. Soc., 38, 39-51. 
GOSLING L.M., BAKER S.J., 1991. Family Myocastoridae in CORBET G.B. and HARRIS S., The Handbook of British mammals, Third Edition, 267-298, Blackwell, Oxford.

KAUHALA K., HELLE E., 1995. Population ecology of the raccoon dog in Finland - a synthesis. Wildife Biology, 1, (1), 4-9.

LAIDLER L., 1982. Otters in Britain. David and Charles, London, $200 \mathrm{p}$.

LINSCOMBE G., KINLER N., AULERICH R.J., 1982. Mink in CHAPMAN J.A. and FELDHAMER G.A., Wild Mammals of North America, 629-643, The Johns Hopkins University Press, Baltimore.

MACDONALD S.M., MASON C.F., 1994. Statut et besoins de conservation de la loutre (Lutra lutra) dans le Paléarctique occidental. Sauvegarde de la Nature, 67, Conseil de l'Europe, Strasbourg, $54 \mathrm{p}$.

MACDONALD D., 1995. European Mammals. Harper Collins, London, 352 p.

MAIZERET C., 1990. Le vison d'Amérique. Encyclopédie des Carnivores de France, 14, SFEPM, Paris, $19-44$.

MAURIN H., 1997. L'homme et les mammifères de France métropolitaine : évolution historique et introductions d'espèces dans les milieux humides et aquatiques. Bull. Fr. Pêche Piscic., 344-345.

MAURIN H., HAFFNER P., KEITH P., 1994. Bilan des introductions et réintroductions de vertébrés sauvages en France métropolitaine depuis le début du siècle. Colloques d'histoire des connaissances zoologiques, Tome 5. Des animaux introduits par l'homme dans la faune de l'Europe, Université de Liège, 79-104.

MICOL T., 1990. Le ragondin. La Défense des Végétaux, 260-261, 40-45.

MICOL T., 1991. Statut et gestion des populations de ragondin dans leur pays d'introduction. Bulletin Technique d'Information, 3, nouvelle série, 2-4.

MOUTOU F., 1989. Les zoonoses transmises par les rongeurs anthropophiles. La Défense des Végétaux, 255-256, 43-47.

MOUTOU F., 1990. Maladies transmissibles à l'homme et au bétail par les rongeurs. La Défense des Végétaux, 260-261, 64-67.

MOUTOU F., 1994. Déplacements d'espèces animales par l'homme : conséquences écologiques et sanitaires. Anthropozoologica, 19, 3-8.

PERRY Jr H.R., 1982. Muskrats in CHAPMAN J.A. and FELDHAMER G.A., Wild Mammals of North America, 282-325, The Johns Hopkins University Press, Baltimore.

PIQUEMAL J.P., TOUSSAINT M., 1991a. Le ragondin, acquis du groupe de travail ragondins de l'Ouest de la France. Bulletin Technique d'Information, 3, nouvelle série, 5-13.

PIQUEMAL J.P., TOUSSAINT M., 1991b. Le rat musqué. Bulletin Technique d'Information, 3, nouvelle série, 14-16.

SAINT-GIRONS M.C., 1994. Le vison sauvage (Mustela lutreola) en Europe. Sauvegarde de la Nature, 54, Conseil de l'Europe, Strasbourg, $41 \mathrm{p}$.

SAND E., KLEIN F., 1995. Les populations de daim, de cerf sika et d'hydropote en France. B. M. O.N.C., 205, 32-39.

TORRENCE M.E., JENKINS S.R., GLICKMAN L.T., 1992. Epidemiology of raccoon rabies in Virginia 1984 to 1989. J. Wildl. Dis., 28, 369-376.

WILSON D.E., REEDER D.A., 1993. Mammals species of the World, $2^{\text {nd }}$ Edition. Smithsonian Institution Press, Washington and London, $1206 \mathrm{p}$. 